\title{
A PLANE VERTICAL SUBMERGED BARRIER IN SURFACE WATER WAVES
}

\author{
U. BASU and B.N. MANDAL \\ Department of Applied Mathematics \\ University of Calcutta \\ 92 A.P.C. Road, Calcutta-700009 \\ INDIA
}

(Received October 3, 1986 and in revised form October 27, 1986)

ABSTRACT. By a simple application of Green's Integral theorem, amplitude of the radiated waves at infinity due to a line source in the presence of a fixed vertical plane barrier completely submerged in deep water is obtained.

KEYS WORDS AND PHRASES. Line source, submerged barrier, velocity potential, Green's theorem, amplitude of radiated waves.

1980 AMS SUBJECT CLASSIFICATION CODE. $76 B$.

1. INTRODUCTION.

In the linearized theory of water waves under the assumption of irrotational motion the velocity potential due to a harmonically oscillating line source present in deep water was given by Thorne [1] in course of a systematic survey of different types of singularities present in the water. The radiation of waves due to this line source is in general affected by an obstacle present in the water. However, when the obstacle is in the form of a submerged long horizontal circular cylinder the radiated waves are uneffected (cf. Evans [2]). When the obstacle is in the form of a fixed vertical plate partially immersed in deep water, the complex amplitudes of the radiated waves at infinity were obtained by Evans [3] after using the Green's integral theorem in the fluid region to the velocity potential appropriate to the problem and the known potential function for the corresponding scattering problem due to a normally incident wave train given by Ursell [4].

In the present note we consider the problem of radiation of waves due to a line source in the presence of a submerged fixed vertical plane barrier. This is complimentary to the problem of the immersed barrier considered earlier by Evans [3]. The velocity potential for the corresponding scattering problem due to a normally incident wave train was obtained by many authors employing different techniques, the earliest by Dean [5] and the latest by Goswani [6]. Here we apply the Green's integral theorem in the fluid region using the known potential function for the corresponding scattering problem as given by Goswami [6] to obtain the complex amplitudes of the radiated waves at infinity.

2. STATEMENT AND FORMULATION OF THE PROBLEM.

We consider an infinitely long plane vertical barrier submerged in deep water and 
use a co-ordinate system in which the y-axis is taken vertically downwards, the mean free surface (FS) is the plane $y=0$ and the position of the barrier is given by $\mathrm{x}=0, \mathrm{a}<\mathrm{y}<\infty$. Let a harmonically oscillating line source of unit strength and of circular frequency $\omega$ be present at the point $(\xi, \eta), \eta>0$. It is assumed that the fluid is incompressible, inviscid and the motion is irrotational so that a velocity potential exists. As the motion is produced by the harmonically oscillating line source it can be described by $\operatorname{Re}\{\Phi(x, y) \exp (-i \omega t)\}$ whence, assuming the linearized theory of water waves, $\Phi(x, y)$ satisfies

$$
\begin{aligned}
& \nabla^{2} \Phi=0 \text { in the fluid region except at }(\xi, n), \\
& K \Phi+\frac{\partial \Phi}{\partial y}=0 \text { on } y=0
\end{aligned}
$$

where $\mathrm{K}=\omega^{2} / \mathrm{g}, \mathrm{g}$ being the gravity. (2.1) is the equation of continuity and (2.2) is the linearized FS condition. Also, near the edge of the barrier at $(0, a),|\nabla \Phi|$ has an integrable singularity which is usually expressed as

$$
\left\{x^{2}+(y-a)^{2}\right\}^{1 / 2}|\nabla \Phi| \rightarrow 0 \text { as }(x, y) \rightarrow(0, a)
$$

(2.3) is the so-called edge condition to be satisfied near the edge of a submerged barrier. As $|x| \rightarrow \infty, \Phi$ w111 represent outgoing waves the amplitudes of which (as $x \rightarrow+\infty$ and $x \rightarrow-\infty$ ) are required to be found out.

Let $\mathrm{G}(\mathrm{x}, \mathrm{y} ; \xi, \eta)$ denote the potential due to the line source of unit strength situated as $(\xi, \eta)$ in the absence of the submerged barrier. Then $G$ satisfies the Laplace's equation in the region $y \geqq 0$ except at $(\xi, n)$, the FS condition $(2.2)$, the condition that it behaves as an outgoing wave as $|x-\xi| \rightarrow \infty$, and $G,|\nabla G| \rightarrow 0$ as $\mathrm{y} \rightarrow \infty$. It is known from [1] that

$$
\begin{aligned}
G(x, y ; \xi, n) & =-2 \int_{0}^{\infty} \frac{(k \cos k n-k \sin k n)(k \cos k y-k \sin k y)}{k\left(k^{2}+k^{2}\right)} \\
& x \exp (-k|x-\xi|) d k-2 \pi i \exp \{-k(y+n)+i k|x-\xi|\}
\end{aligned}
$$

Now the potential function $\Phi$ can be expressed as

$$
\Phi=G+\phi
$$

where $\phi(x, y)$ can be regarded as the correction to $G$. due to the presence of the barrier. Since the barrier is fixed

$$
\frac{\partial \Phi}{\partial x}=0 \text { on } x=0, a<y<\infty
$$

so that

$$
\frac{\partial \phi}{\partial x}=-\frac{\partial G(0, y ; \xi, \eta)}{\partial x}=f(y) \text {, say, on } x=0 \text {, a }<y<\infty \text {. }
$$

Let

$$
\Phi \sim \mathrm{B}^{ \pm}(\xi, n) \exp (\mathrm{iK}|\mathrm{x}|-\mathrm{Ky}) \text { as } \mathrm{x} \rightarrow \pm \infty
$$


where $B^{ \pm}$are the complex amplitudes of the radiated waves at positive and negative infinity respectively and are to be determined. Thus $\phi$ satisfies (2.1), (2.2), (2.6). Because of (2.6), $\phi$ is odd in $x$. Again, far away from the barrier and also from the source, the waves travel away from the barrier so that

$$
\phi \sim \pm A \exp (i K|x|-y) \text { as } x \rightarrow \pm \infty
$$

where $A$ is the complex amplitude (unknown) of the scattered field. In addition

$$
\phi,|\nabla \phi| \rightarrow 0 \text { as } \mathrm{y} \rightarrow \infty \text {. }
$$

Also, because of $(2.3)$, since $|\nabla G|$ is not singular near $(0, a)$,

$$
\left\{x^{2}+(y-a)^{2}\right\}^{1 / 2}|\nabla \phi| \rightarrow 0 \text { as }(x, y) \rightarrow(0, a) \text {. }
$$

3. SOLUTION OF THE PROBLEM.

Let $\Psi(\mathrm{x}, \mathrm{y})$ denote the velocity potential for the corresponding scattering problem or more specifically, it is the potential due to a progressive wave $\exp (\mathrm{iKx}-\mathrm{Ky})$ incident upon the barrier from negative infinity. The explicit form for $\Psi(x, y)$ is given by Goswami [6] (also it is apparent from [4]) as

$$
\begin{aligned}
\Psi(x, y) & =\frac{1}{\Delta(K a)}\left[\pi I_{0}(K a) \exp (i K x-k Y)-i \int_{0}^{\infty} \frac{J_{0}(k a)}{K^{2}+k^{2}}\right. \\
& x(k \cos k y-K \sin k y) \exp (i k x) d k], \text { for } x>0
\end{aligned}
$$

and

$$
\begin{aligned}
\Psi(x, y) & =\exp (i K x-K y)-\frac{1}{\Delta(K a)}\left[i K_{0}(K a) \exp (-i K x-K y)\right. \\
-i & \left.\int_{0}^{\infty} \frac{J_{0}(k a)}{K^{2}+k^{2}}(k \cos k y-K \sin k y) \exp (k x) d k\right], \text { for } x<0
\end{aligned}
$$

where $\Delta(\mathrm{Ka})=\pi \mathrm{I}_{\mathrm{o}}(\mathrm{Ka})-i \mathrm{~K}_{\mathrm{o}}(\mathrm{Ka})$,

$I_{0}, K_{o}$ being the modified Bessel functions of order zero.

We now apply the Green's integral theorem to the harmonic functions $\phi$, $\psi$ within the region bounded by the 1ines, $y=0,|x| \leqq X ; x=X, 0 \leqq y \leqq Y ; y=Y, 0<x \leqq X ;$ $\mathrm{x}=0+, \mathrm{a} \leqq \mathrm{y} \leqq \mathrm{Y} ; \mathrm{x}=0-, \mathrm{a} \leqq \mathrm{y} \leqq \mathrm{Y} ; \mathrm{y}=\mathrm{Y},-\mathrm{X} \leqq \mathrm{x}<0 ; \mathrm{x}=-\mathrm{X}, 0 \leqq \mathrm{y} \leqq \mathrm{Y}$. Let $C$ be the contour consisting of these lines, then we have

$$
\int_{C}\left(\phi \frac{\partial \Psi}{\partial \mathrm{n}}-\Psi \frac{\partial \phi}{\partial \mathrm{n}}\right) \mathrm{ds}=0
$$

where $\mathrm{n}$ is the outward normal to the line element ds. Conditions (2.2) and (2.9) ensure that there is no contribution to (3.4) from the FS or from the line $y=Y$, $|X| \leqq X$, as $Y \rightarrow \infty$. In addition, because both $\phi$ and $\psi$ describe outgoing waves as $x \rightarrow+\infty$, there is no contribution from $x=x, 0 \leqq y \leqq Y$ as $X \rightarrow \infty$. The only contribution arises from the line integral around the barrier and from the incoming wave in $\Psi(\mathrm{x}, \mathrm{y})$. Combining with the outgoing wave from $\phi(\mathrm{x}, \mathrm{y})$ for $\mathrm{x}=-\mathrm{x}$, $0 \leqq y \leqq Y$ as $X, Y \rightarrow \infty$, we obtain

$$
A=i \int_{0}^{\infty} g(y) f(y) d y
$$


where

$$
\mathrm{g}(\mathrm{y})=\Psi(0+, \mathrm{y})-\Psi(0-, \mathrm{y}) \quad \mathrm{a}<\mathrm{y}<\infty
$$

denotes the jump in $\Psi$ across the barrier so that from (3.1) and (3.2)

$$
g(y)=\frac{2 i}{\Delta(K a)}\left[K_{0}(K a) \exp (-K y)-\int_{0}^{\infty} \frac{J_{0}(k a)}{K^{2}+k^{2}}(k \cos k y-K \sin k y) d k\right] \text {. }
$$

To simplify $g(y)$ we note that

$$
\begin{aligned}
K g(y)+g^{\prime}(y) & =\frac{2 i}{\Delta} \int_{0}^{\infty} J_{0}(k a) \sin k y d k \\
& =\frac{2 i}{\Delta}\left\{\begin{array}{cc}
0, & 0<y \leqq a \\
\left(y^{2}-a^{2}\right)^{-1 / 2}, & y>a
\end{array}\right.
\end{aligned}
$$

Thus

$$
g(y)=\left\{\begin{array}{cll}
0 & , 0<y \leqq a, \\
\frac{2 i}{\Delta} & \exp (-K y) & \int_{a}^{y} \frac{\exp (k v)}{\left(v^{2}-a^{2}\right)^{1 / 2}} d v, y>a
\end{array}\right.
$$

since $g(a)=0$.

Now

$$
\int_{a}^{\infty} g(y) f(y) d y=-\frac{2 i}{\Delta} \int_{a}^{\infty} \frac{\exp (K y) h(y)}{\left(y^{2}-a^{2}\right)^{1 / 2}} d y
$$

where

$$
h(y)=\int_{\infty}^{y} f(u) \exp (-K u) d u
$$

Thus

$$
A=\frac{2}{\Delta} \int_{a}^{\infty} \frac{s(y)}{\left(y^{2}-a^{2}\right)^{1 / 2}} d y
$$

where

$$
s(y)=\exp (K y) \int_{\infty}^{a} f(u) \exp (-K u) d u .
$$

But by (2.4) and (2.6) we obtain

$$
\begin{aligned}
& s(y)=2 \operatorname{sgn} \xi\left[\int_{0}^{\infty} \frac{\sin k y(k \cos k n-k \sin k n)}{k^{2}+k^{2}} \exp (-k|\xi|) d k\right. \\
& -\pi \exp (i K|\xi|-K n-K y)]
\end{aligned}
$$

Substitution into (3.6) gives, after simplification

$$
\begin{aligned}
A & =\frac{2 \pi}{\Delta} \operatorname{sgn} \xi\left[\int_{0}^{\infty} \frac{J_{0}(k a)(k \cos k n-K \sin k n)}{K^{2}+k^{2}} \exp (-k|\xi|) d k\right. \\
& \left.-K_{0}(K a) \exp (i K|\xi|-K n)\right] .
\end{aligned}
$$

This gives the complex amplitude of the scattered field. Now from $(2.5),(2.4),(2.8)$ and (3.9) it follows that as $x \rightarrow+\infty$ 


$$
\begin{aligned}
\Phi(\mathrm{x}, \mathrm{y} ; \xi, \eta)=G_{0}+ & \phi \\
& \sim-\frac{2 \pi i}{\Delta} \exp (i K x-K y)\left[\Delta \exp (-i K \xi-K \eta)-i K_{0}(K a) \operatorname{sgn} \xi \exp (i K|\xi|-K \eta)\right. \\
& \left.+i \operatorname{sgn} \xi \int_{0}^{\infty} \frac{J_{0}(k a)(k \cos k n-K \sin k n)}{K^{2}+k^{2}} \exp (-k|\xi|) d k\right]
\end{aligned}
$$

as $\mathrm{x} \rightarrow+\infty$, and while as $\mathrm{x} \rightarrow-\infty$,

$$
\begin{gathered}
\Phi \sim-\frac{2 \pi i}{\Delta} \exp (-i K x-K y)\left[\Delta \exp (i K \xi-K n)+i K_{0}(K a) \operatorname{sgn} \xi \exp (i K|\xi|-K n)\right. \\
\left.+i \operatorname{sgn} \xi \int_{0}^{\infty} \frac{J_{0}(k a)(k \cos k n-K \sin k n)}{K^{2}+k^{2}} \exp (-k|\xi|) d k\right] .
\end{gathered}
$$

Thus from $(2.7),(3.1),(3.2),(3.10)$ and $(3.11)$ we obtain

$$
\left.\begin{array}{l}
\mathrm{B}^{+}(\xi, \eta)=-2 \pi i \exp (-i K \xi-K n)+A=-2 \pi i \Psi(\xi, n) \\
\mathrm{B}^{-}(\xi, \eta)=-2 \pi i \exp (i K \xi-K \eta)-A=-2 \pi i \Psi(-\xi, n) .
\end{array}\right\}
$$

An immediate consequence is

$$
\mathrm{B}^{+}(\xi, n)+\mathrm{B}^{-}(\xi, n)=4 \pi i \exp (-\mathrm{Kn}) \cos \mathrm{K} \xi .
$$

For high frequency waves $(\mathrm{Ka} \rightarrow \infty) \mathrm{A} \rightarrow 0$ so that

$$
\mathrm{B}^{ \pm}(\xi, n) \rightarrow-2 \pi i \exp (\mp i K \xi-K n)
$$

which shows that there is no effect of the barrier on the radiated waves from the source. This is to be expected as in this case the waves are confined within a thin layer below the FS so that the presence of the barrier is not felt by the waves for sufficiently large wave number.

Again when the source is directly above the barrier, so that $\xi=0, \eta<a$, then

$$
\Psi(0, n)=\exp (-K n)
$$

as

$$
\int_{0}^{\infty} \frac{J_{0}(K a)(k \cos k n-K \sin k n)}{K^{2}+k^{2}} d k=K_{0}(K a) \exp (-K \eta), \eta<a
$$

and we obtain from (3.12)

$$
B^{+}(0, n)=B^{-}(0, n)=-2 \pi 1 \exp (-K n)
$$

and the barrier has no effect on the source.

When $K \xi$ is an odd multiple of $\pi / 2$ we conclude from (3.12) that "the wave amplitudes on either infinity are same, the surface elevations being exactly $180^{\circ}$ out of phase with each other". Similar conclusion was also arrived at by Evans [3] while considering the immersed barrier problem.

\section{REFERENCES}

1. THORNE, R.C. Multipole Expansion in the Theory of Surface Waves, Proc. Camp. Phil. Soc. 49(1953), 701-716.

2. EVANS, D.V. A Note on the Transparency of a Submerged Circular Cylinder to the Waves Radiated by a Pulsating Line Source, IMA J. App1. Maths. 33(1984), 105-107. 
3. EVANS, D.V. A Note on the Waves Produced by the Small Oscillations of a Partially Immersed Vertical Plate, J. Inst. Maths. Applics. 17 (1976), 135-140.

4. URSELL, F. The Effect of a Fixed Vertical Barrier on Surface Waves in Deep Water, Proc. Camp. Phil. Soc. 43(1947), 374-382.

5. DEAN, W.R. On the Reflexion of Surface Waves by a Submerged Plane Barrier, Proc. Camp. Phil. Soc. 41 (1945), 231-238.

6. GOSWANI, S.K. A Note on the Problem of Scattering of Surface Waves by a Submerged Fixed Vertical Barrier, ZAMM 62(1982), 637-639. 


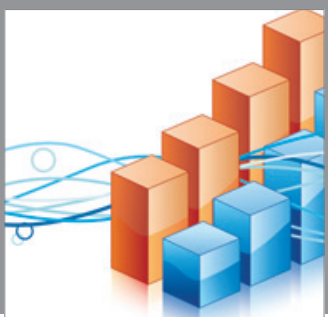

Advances in

Operations Research

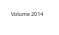

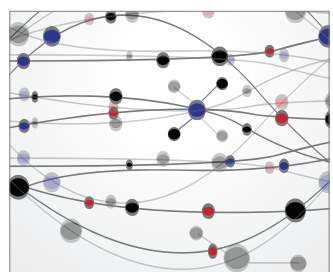

\section{The Scientific} World Journal
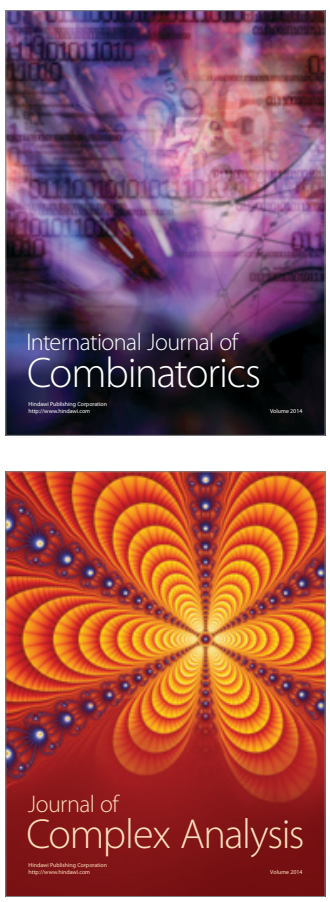

International Journal of

Mathematics and

Mathematical

Sciences
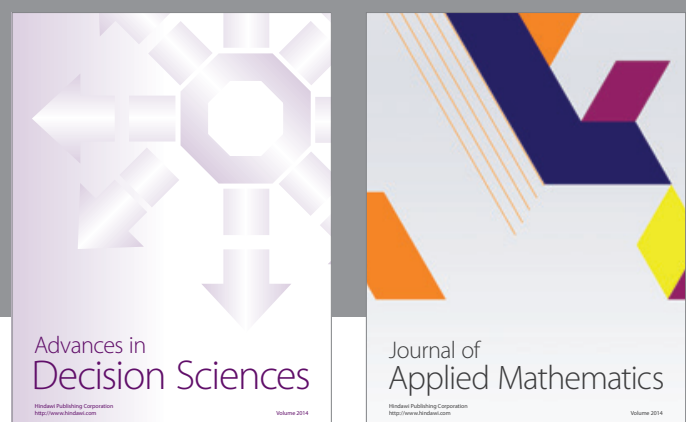

Journal of

Applied Mathematics
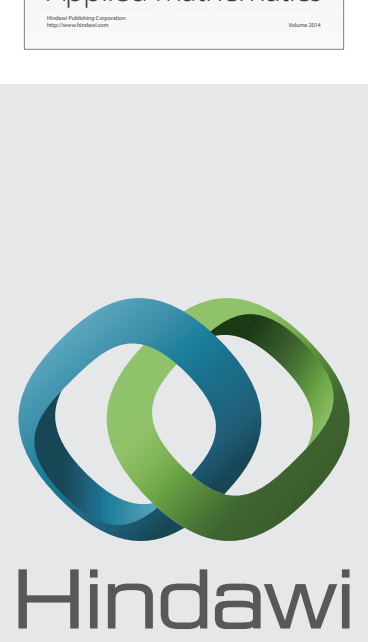

Submit your manuscripts at http://www.hindawi.com
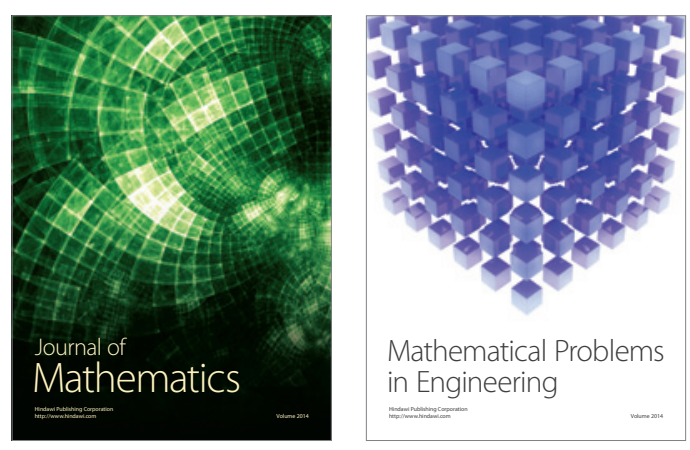

Mathematical Problems in Engineering
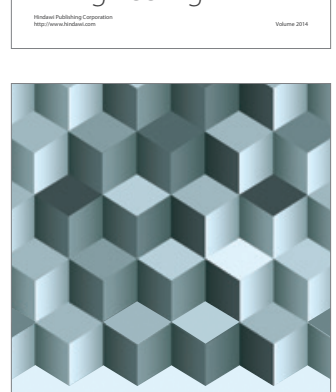

Journal of

Function Spaces
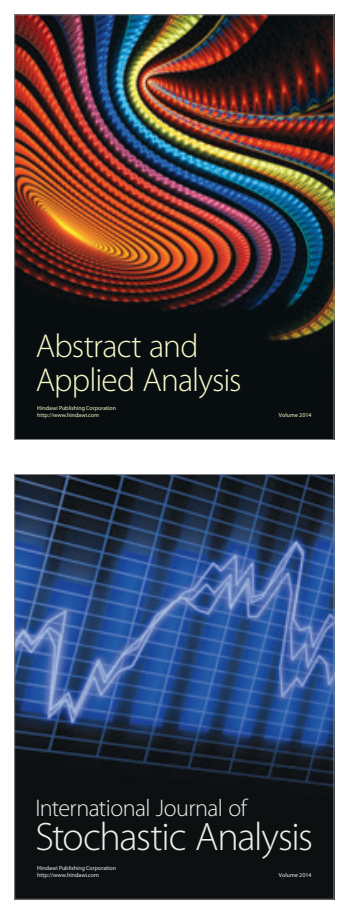

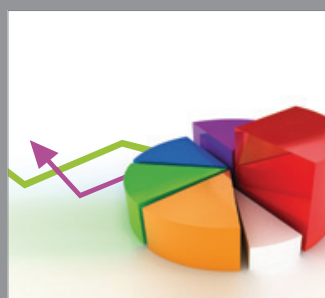

ournal of

Probability and Statistics

Promensencen
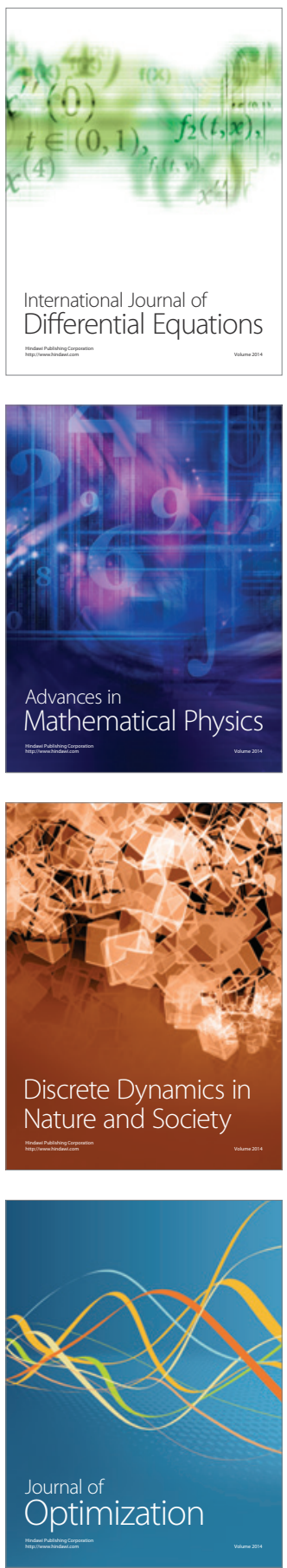